pediatric examination described by Pine et al ( $L$ Am Acad Child Adolesc Psychiatry 1996;35:509-515), and externalizing and internalizing psychopathology was examined in 56 boys, $8.5(+/-1.5)$ years old, from a highrisk sample treated at the New York State Psychiatric Institute, NY. Soft sign measurements, based on 64 observations of 11 motor tasks performed in 20 minutes, included motor slowness, accuracy, abnormal movements, and smoothness. The results of this soft sign exam and correlations with psychiatric symptoms were stable over a 1-year period. Symptoms of externalizing disorders (ODD and CD) and internalizing disorders (anxiety, phobias, depression or dysthymia) correlated with impaired performance on the soft sign examination. An association between soft signs and ADHD was nonsignificant. (Pine DS, Wasserman GA, Fried JE, Parides M, Shaffer D. Neurological soft signs: one-year stability and relationship to psychiatric symptoms in boys. I Am Acad Child Adolesc Psychiatry Nov 1997;36:1579-1586). (Respond: Dr Daniel S Pine, New York State Psychiatric Institute, Unit 78, 722 W 168th Street, New York, NY 10032).

COMMENT. Childhood subtle impairments in motor performance, involving motor speed, accuracy, and fluency, may be exhibited by children with $\mathrm{ODD} / \mathrm{CD}$ or anxiety and depression disorders, in the absence of specific abnormal neurological signs. The authors and others attribute these soft neurological signs and associated psychiatric symptoms to basal ganglia dysfunction. The lack of significant association between ADHD and soft signs, as measured by the Pine method, is surprising. The demonstration of soft signs in a neurological examination may be a risk factor for childhood onset psychiatric symptoms, and for ADHD. (see Millichap JG. Progress in Pediatric Neurology III, Chicago, PNB Publishers, 1997;p196).

\title{
FETAL ALCOHOL EXPOSURE AND ATTENTION DEFICITS
}

Twenty six children of mothers who abused alcohol during pregnancies were followed throughout childhood and were examined at 11 to 14 years of age for neuropsychiatric, psychological, and social problems in the Sahlgren University Hospital, Goteborg, Sweden. Of 24 seen at follow-up, 10 had ADHD, 2 had low normal intelligence and Asperger syndrome, and one had mild mental retardation, spastic diplegia, and an autistic-like condition. Seventeen required special education, six for mental retardation, and only 7 attended regular schools without support services. Specific learning disabilities involved math, visual perception, short-term memory, and attention. Sixteen were in foster homes. The severity of the neuropsychiatric disorder was correlated with the degree of alcohol exposure in utero. (Aronson M, Hagberg B, Gillberg C. Attention deficits and autistic spectrum problems in children exposed to alcohol during gestation: a follow-up study. Dev Med Child Neurol Sept 1997;39:583-587). (Respond: Marita Aronson PhD, Department of Pediatrics, Sahlgren University Hospital, Goteborg, Sweden).

COMMENT. The majority of children exposed to alcohol abuse in utero have attention deficits, poor motor control, and learning disorders. An autistic spectrum disorder, including Asperger syndrome, may occur in a minority. The severity of the neuropsychiatric disorders is correlated with the degree and duration of alcohol abuse. Children whose mothers discontinue alcohol consumption by the 12th week of gestation develop normally and are not likely to have learning difficulties in school. Biological, not psychosocial factors, are responsible for the neuropsychiatric disorders in fetal alcohol 
syndrome.

Fetal valproate syndrome (FVS) and autism are reported in a 5 year-old boy exposed to valproic acid (VPA) in utero. (Williams PG, Hersh JH. Dev Med Child Neurol Sept 1997;39:632-634). The mother had taken VPA (500 mg 4 xday) for 2 to 3 years before and through the 5 th month of pregnancy. The infant's speech and language were delayed, and he communicated primarily by gestures. His head circumference was $55.5 \mathrm{~cm}$ (>95th centile), the forehead high and bossed, the nasal bridge flat, and the upper lip thin. Neurologic abnormalities included hypotonia, hyperreflexia, and ankle clonus. This is the second report of FVS manifested by autism.

\section{LEARNING DISABILITY SUBTYPES IN NEUROFIBROMATOSIS 1}

An analysis of neuropsychological data for 72 children, aged 6 to 18 years, with neurofibromatosis 1 (NF-1) and academic deficiencies is reported from the University of Texas MD Anderson Cancer Center, Houston, TX. Patients treated with cranial irradiation $(6 \%)$ were excluded. Those with MRI areas of hyperintensity were not excluded. The IQs of the groups analyzed ranged from 78 to 107 , and outlier groups ( $<5$ patients) with above average IQs or the mentally deficient were excluded. Three groups of academic underachievers were identified: 1) neuropsychologically normal (39\%); 2) a group with general academic deficiencies (47\%); and 3) a group with visuospatialconstructional and fine motor coordination deficiencies, and without language deficits (14\%). The low incidence of visuospatial deficiencies and absence of a group with pure language deficits were remarkable. (Brewer VR, Moore BD III, Hiscock M. Learning disability subtypes in children with neurofibromatosis. Irnl of Learning Disabilities Sept/Oct 1997;30:521-533). (Respond: Bartlett D Moore III, Division of Pediatrics (Box 87), UTMD Anderson Cancer Center, 1515 Holcombe Blvd, Houston, TX 77030).

COMMENT. By using cluster analysis and exclusion of outlier groups with above average or retarded IQ scores, children with neurofibromatosis 1 who are academic underachievers may fall into one of three groups psychologically. Approximately 40\% test normal, 50\% have general learning disabilities, and $14 \%$ have visuospatial and motor coordination problems.

Correlations of cognitive impairments with MRI abnormalities and unidentified bright objects (UBOs) are of interest. A relation between the number or location of UBOs on MRI and cognitive deficits in children with NF1 have been reported. The University of Texas study group (Moore et al, 1996) found location important, UBO hyperintensities in the thalamus correlated with a lowered IQ whereas the Johns Hopkins group (Denckla et al, 1996) found the number of locations occupied by UBOs accounted for the lowering of IQ. (See Progress in Pediatric Neurology III, PNB Publishers, 1997; p291-294, p441-442, for articles and commentary on IQ and MRI findings in NF-1).

\section{SEIZURE DISORDERS}

\section{GENETICS OF FAMILIAL INFANTILE CONVULSIONS}

Four families from northwestern France with autosomal dominant benign infantile convulsions and paroxysmal choreoathetosis were studied genetically by linkage analysis at the Wellcome Trust Centre for Human Genetics, University of Oxford, UK, and at Genetic Clinics in Amiens, France. Linkage for the disease gene was found in the pericentromeric region of 